\title{
Evaluating the Prevalence and the Risk Factors of Gram-Negative Multi-Drug Resistant Bacteria in Eastern Saudi Arabia
}

\author{
Aisha S Al Hamdan', Amal A Alghamdi $\mathbb{D}^{2}$, Ghada F Alyousif', Fatma A Hamza $\mathbb{D}^{2}$, Marwa M Shafey $\mathbb{D}^{2}$, \\ Aisha M AlAmri $\mathbb{D}^{3}$, Abdulaziz A Sunki ${ }^{4}$ \\ 'Department of Infection Control and Prevention, Security Forces Hospital, Dammam, Saudi Arabia; ${ }^{2}$ Department of Family and Community \\ Medicine, Imam Abdulrahman Bin Faisal University, Dammam, Saudi Arabia; ${ }^{3}$ Department of Clinical Laboratory Sciences, Imam Abdulrahman Bin \\ Faisal University, Dammam, Saudi Arabia; ${ }^{4}$ General Laboratory Division, Microbiology Unit, Johns Hopkins Aramco Healthcare (JHAH), Dhahran, \\ Saudi Arabia
}

Correspondence: Aisha S Al Hamdan, Department of Infection Control and Prevention, Security Forces Hospital, Dammam, Saudi Arabia, Tel+966/38104863, Email aalhamdan@sfhd.med.sa

Purpose: Multidrug-resistant organisms (MDROs) among Gram-negative bacteria (GNB) are a major public health concern worldwide, which can even lead to death. This study was conducted to determine the prevalence of MDROs among isolated GNB in the Security Forces Hospital Dammam (SFHD) and identify its associated risk factors.

Materials and Methods: A cross-sectional study was performed on the most commonly isolated GNB in SFHD, Acinetobacter spp., Enterobacter spp., Escherichia coli (E. coli), Klebsiella pneumoniae, Proteus spp., and Pseudomonas aeruginosa, of non-duplicated clinical samples collected from all hospital units throughout the period from January 2017 to December 2018. Data were collected retrospectively from patients' medical records, and analyses were conducted using the chi-square test and logistic regression models. Results: Of the 1508 GNB included in the study, 969 were multidrug-resistant (MDR; 64.3\%). The most commonly identified multidrug-resistant GNB (MDR-GNB) were found in female patients $(66.4 \%)$ and those aged between 20 and 29 years (21.8\%). Urine samples had the highest number of isolated GNB (926 of a total of 1508, 61.4\%), and E. coli isolates (53.8\%) were the most frequently isolated GNB. Enterobacter spp. had the highest rate of multidrug resistance during the 2 years (64 out of 74, 86.5\%). Mechanical ventilation for three or more calendar days was a significant direct risk factor for the development of MDR-GNB (odds ratio [OR]: $2.600,95 \%$ confidence interval [CI]: 1.124-6.012, $\mathrm{P}=0.025)$.

Conclusion: Multidrug resistance is common among GNBs in SFHD. Antimicrobial stewardship programs in hospitals should be supported and implemented. Medical and public awareness of antibiotic use is another significant way to decrease the burden of MDR.

Keywords: antimicrobial resistance, hospital-acquired pathogens, infectious diseases

\section{Introduction}

The widespread of multidrug-resistant organisms (MDROs) represents a global concern documented worldwide. ${ }^{1}$ MDROs are recognized as hospital-acquired pathogens linked to prolonged hospitalization and increased morbidity and mortality, which represents a significant economic burden on healthcare systems. ${ }^{1}$ Gram-negative bacteria (GNB) are frequently responsible for serious conditions such as pneumonia, meningitis, and sepsis. ${ }^{2}$ The emergence of multidrug resistance among Gram-negative organisms has become a medical challenge to treat infectious diseases because of limited therapeutic options. ${ }^{3}$

In the United States of America (US), antimicrobial resistance exerts an additional 20 billion dollar on healthcare costs. ${ }^{5}$ Globally, antimicrobial resistance kills more than 700,000 individuals annually. Furthermore, it has been estimated that the annual fatalities attributed to antimicrobial resistance (AMR) will increase up to 10 million by 
2050, costing 100 trillion USD. ${ }^{4}$ The rapid dissemination of multidrug resistance depends on several factors such as frequent hospitalizations, post-surgical operations, use of a Foley catheter (FC), ventilator or central line, previous antibiotic therapy, and diminished compliance to infection control measures. ${ }^{6}$ The majority of these MDROs possess carbapenem-hydrolyzing $\beta$-lactamase enzymes such as class A, B, and D $\beta$-lactamases. ${ }^{6}$ On the other hand, the presence of intrinsic, chromosomal $\beta$-lactam resistance such as the case with species like Enterobacter spp. and Citrobacter spp. may complicate the picture of antibiotics resistance especially when coupled with other resistance mechanisms such as reduced membrane permeability and active efflux pumps. ${ }^{7}$ Since majority of the former resistant genes are carried on mobile elements like plasmids and conjugative transposons, this will ease the vertical transmission of resistance across other GNB occupying the same setting. Along with their increased antibiotic resistance profile, GNB are known to produce biofilms on biotic and abiotic surfaces such as catheters and medical equipment. ${ }^{8,9}$ Significant complications of MDROs include transmitting MDROs between individuals resulting in outbreaks, serious infections with prolonged length of hospital stay, increase in morbidity and mortality, and extensive use of hospital resources such as the need for private isolation room. ${ }^{4,10,11}$

In Saudi Arabia, most studies on the epidemiology of MDROs have been conducted in many regions; however, evaluating the risk factors that lead to emergence of MDROs and their transmission needs to be further explored. ${ }^{12,13}$ In addition, most of the published studies targeted intensive care unit (ICU) patients and/or hospital-admitted patients; however, very few studies have focused on patients visiting the outpatient or emergency department services, which might reflect the rate of MDROs within the community setting. ${ }^{11,13}$ Continuous monitoring of risk factors contributing to infection with MDROs facilitates appropriate utilization of antibiotics, thus minimizing the selection of MDRO pathogens that resist the most advanced antimicrobial agents. In this context, our study will help fill the gap by determining the prevalence of MDROs among isolated GNB in various Security Forces Hospital Dammam (SFHD) departments during 2017 and 2018 and identifying the associated risk factors.

\section{Materials and Methods}

\section{Identification of Bacterial Isolates}

The specimens obtained in the diagnostic microbiology laboratory at SFHD were inoculated on blood and MacConkey agar plates (SPML, Riyadh, KSA) and incubated overnight at $37^{\circ} \mathrm{C}$. Colonies suspected to cause the infections were tested to identify the isolates using the VITEK 2 system (bioMérieux Inc., Durham, NC, USA) using the identification card for Gram-negative strains (ID-GNB) following the manufacturer's recommendations. MDR strains were categorized according to guidelines that dictate the classification of drug-resistant bacteria (non-susceptible [intermediate or resistant] to $\geq 1$ agent in $\geq 3$ antimicrobial categories). ${ }^{14}$

Antibiotic susceptibility testing (AST) was performed using the Vitek 2 system, following the Clinical and Laboratory Standards Institute (CLSI) guidelines for interpretation of susceptibility testing result. ${ }^{15}$ Control strains, E. coli ATCC 25922, Klebsiella pneumoniae ATCC 700603, and Pseudomonas aeruginosa ATCC 27853, were included in every antibiotic susceptibility testing (AST) run.

\section{Inclusion and Exclusion Criteria}

Non-duplicated clinical samples: blood, urine, respiratory (sputum, tracheal aspirate, bronchial lavage and washing, and sinus aspirate), swab (anal, ear, endometrial, eye, genital, groin, placental, pus, skin, throat, vulval, and wound), and others (bile fluid, catheter tip, peritoneal fluid, semen, and tissue) were collected and cultured as routine care from patients. Only the first isolate (GNB) was collected from each patient unless it was collected from different sites.

Patients with incomplete data, missing susceptibility results, or repeated culture results within six months were excluded from this study.

\section{Research Setting}

The study was a cross-sectional design that gathered data from the patients' records and the Diagnostic Microbiology Laboratory entries, retrospectively over the period that extended from the beginning of January 2017 until the end of 
December 2018, conducted in inpatient (INP) units, ICUs, emergency (ER) department, and outpatient (OP) department of SFHD, which is a secondary care hospital with a 176-bed capacity.

The dependent variables were GNB and MDR. The independent variables included sociodemographic features (age and sex), multiple risk factors (location of sample collection, history of admission for SFHD within the past six months, post-operation [past four weeks], use of a Foley's catheter [ $\geq 72 \mathrm{~h}$ ], central line [ $\geq 72 \mathrm{~h}]$, and mechanical ventilator [ $\geq 72$ $\mathrm{h}]$ ), and types of clinical samples.

Body samples of the target population were collected from all age groups (male and female patients).

As we aimed to calculate the prevalence of MDROs among GNB, we first defined the target population $(\mathrm{N}=29,204)$. Next, we determined that the eligible study population satisfied our inclusion and exclusion criteria using the SFHD lab records $(n=1508)$.

The records of patients who provided a clinical sample for culture were reviewed using the hospital information system (HIS) and laboratory information system (LIS). Data were then extracted from the information system, cleaned, and coded using an Excel sheet. Finally, the data were transferred to SPSS (IBM SPSS Statistics 23) for further analysis. The extracted data were divided into two parts. The first part included the organisms and multidrug resistance (yes or no). The second part was the data that included information about sociodemographic features (age [categorized with a 10-year interval] and sex [female or male]), risk factors (location of sample collection (organized into ER (the reference), ICU, INP, and OP], (history of admission in SFHD within the past six months (no (reference) or yes], history of operation during the past four weeks before the onset of GNB (no (the reference) or yes], use of a central line [ $\geq 72 \mathrm{~h}]$ (no [reference] or yes), use of an FC [ $\geq 72 \mathrm{~h}$ ] (no [the reference] or yes), use of a mechanical ventilator [ $\geq 72 \mathrm{~h}$ ] (no [the reference] or yes), and clinical samples (categorized into urine [the reference], blood, respiratory, swab, and others). Therefore, the reference values in our study were selected as the normal value category. However, in the absence of a normal value category, the reference was chosen based on the majority of samples. The prevalence of each type of MDRO among the isolated GNBs was calculated using the following equation:

Period prevalence of MDR bacteria (type $\mathrm{x}$ ) $=$ number of MDROs (type $\mathrm{x}$ )/total number of isolated GNB during the study period (year 1) $\times 100$

The association between independent variables and antibiotic resistance patterns of the isolates was investigated using chi-square analysis, unadjusted logistic regression, and adjusted logistic regression models for age, sex, and location of sample collection. The regression analysis was performed in two steps: first, by including all participants regardless of their specimen sites, and second, by subgrouping our participants according to their source of specimens into the blood sample group to test the association between the central line and MDR-GNB, urine sample group to test Foley's catheter as a risk factor, respiratory sample group to test the risk of using a mechanical ventilator, and wound sample and pus swab group to test the history of surgery during the past four weeks as a risk factor. Statistical significance was set at $\mathrm{p}<0.05$.

\section{Results}

As shown in Figure 1, 1508 isolated bacteria from the most commonly isolated GNB (Acinetobacter spp., Enterobacter spp., E. coli, K. pneumoniae, Proteus spp., and P. aeruginosa) and non-duplicated clinical samples from January 2017 to December 2018 were included in the study analysis.

Of the 1508 GNBs included in the study, 969 (64.3\%) were MDR (Figure 2), and the rate of resistance was higher in the isolated GNBs in 2017 (68.2\%) than in $2018(59.9 \%)\left(\mathrm{X}^{2}=11.186, \mathrm{P}=0.001\right)$. The highest rate of MDR-GNBs was among patients aged between 70 and 79 years ( 86 out of a total of 111, 77.5\%), followed by patients aged 90 years or above (25 out of a total of 33,75.8\%). Resistance was not significantly different between the male and female patients ( $\mathrm{P}$ $=0.084)$. MDR-GNB was high among GNB isolates from samples collected from the INP units (269 out of a total of $408,72.5 \%$ ), ICU ( 80 out of a total of 122, 65.6\%), OP units (198 out of a total of 315, 62.9\%), and ER (395 out of a total of $663,59.6 \%$ ). The highest rate of resistance during the two years was among Enterobacter spp. (64 out of a total of 74; 86.5\%), followed by Proteus spp. (84 out of a total of 116, 72.4\%). The distribution of MDR-GNBs was not significant with respect to the sample type $(\mathrm{P}=0.564)$. The multidrug resistance rate was high in GNB isolated from patients using a central line ( $\geq 72 \mathrm{~h}$ ) ( 29 out of a total of $36,80.6 \%$ ), followed by GNB isolated from patients using 


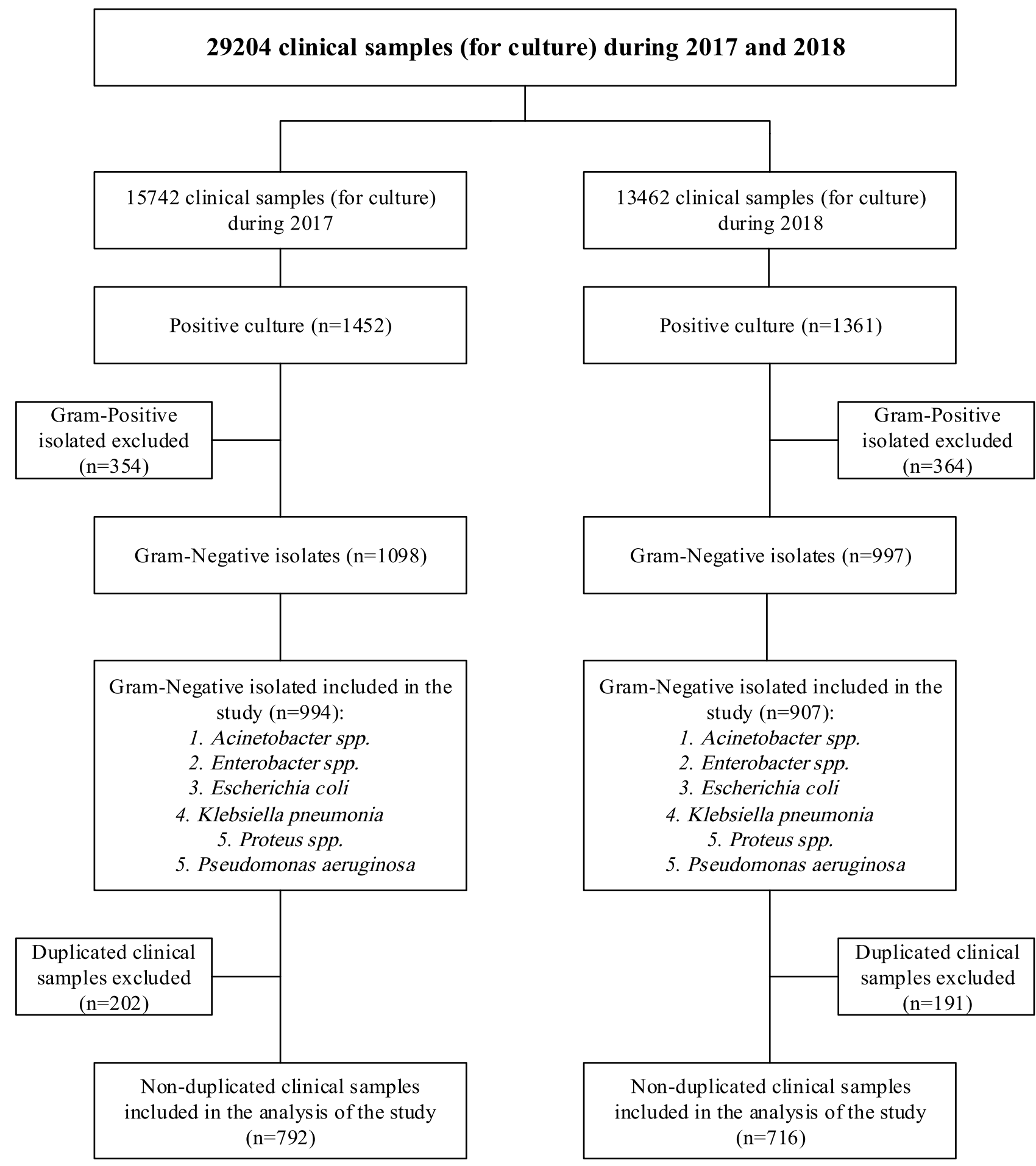

Figure I Flowchart for study design including inclusion/exclusion criteria of isolates.

a mechanical ventilator ( $\geq 72 \mathrm{~h}$ ) (70 out of a total of $91,76.9 \%$ ), and patients with a history of operation before 4 weeks of the onset of GNB (77 out of a total of 102, 75.5\%) (Table 1).

The mean age population was 38.75 years with an SD of 26.750, and the most common age group was between 20 and 29 years (326 out of a total of 1508, 21.6\%). The majority of GNBs were isolated from female patients (1024 of a total of $1508,67.9 \%$ ). Most isolates were from samples collected in the ER (663 out of a total of 1508, 44\%), followed by those in the INP units (408 out of a total of 1508, 27.1\%). Urine samples had the highest number of isolated GNB 


\begin{tabular}{|c|c|c|c|c|c|c|c|c|c|c|c|c|c|c|c|c|c|}
\hline \multicolumn{18}{|c|}{$\%$ Susceptibilty } \\
\hline Species & Year & AMIK & AMP & AMC/CLAV & CZO & FEB & CAZ & CRO & CXM & CIP & LEVo & GENT & IMP & MRP & NFN & PIP/TAZ & TRIMETH \\
\hline \multirow{2}{*}{ E.coli } & 2017 & 91 & 6 & 22 & 11 & 43 & & 35 & 34 & 70 & 40 & 87 & 99 & 99 & 51 & 49 & 49 \\
\hline & 2018 & 71 & 24 & 53 & 50 & 52 & & 50 & 49 & 62 & 84 & 97 & 96 & 61 & 63 & 72 & 47 \\
\hline \multirow{2}{*}{ Klebsiella spp. } & 2017 & 84 & & 35 & 35 & 48 & & 44 & 43 & 82 & 75 & 76 & 99 & 99 & 30 & 68 & 61 \\
\hline & 2018 & 96 & & 16 & 45 & 43 & & 42 & 42 & 78 & 90 & 97 & 97 & 78 & 21 & 56 & 60 \\
\hline \multirow{2}{*}{ Proteus spp. } & 2017 & 79 & 4 & 34 & 36 & 51 & & 45 & 41 & 93 & 87 & 64 & 62 & 62 & & 42 & 47 \\
\hline & 2018 & 93 & 29 & 28 & 25 & 45 & & 39 & 30 & 89 & 68 & 61 & 30 & 89 & & 36 & 59 \\
\hline \multirow{2}{*}{ Enterobacter spp. } & 2017 & 71 & 0 & 0 & 0 & 29 & & 21 & 0 & 93 & 93 & 70 & 100 & 100 & & 29 & 0 \\
\hline & 2018 & 94 & 0 & 0 & 0 & 56 & & 41 & 0 & 91 & 88 & 85 & 74 & 91 & & 47 & 71 \\
\hline \multirow{2}{*}{ Acinetobacter spp. } & 2017 & 42 & & & & 10 & 3 & & & 16 & 16 & 29 & 13 & 13 & & 6 & 19 \\
\hline & 2018 & 82 & & & & 59 & 59 & & & 76 & 82 & 71 & 71 & 76 & & 47 & 88 \\
\hline \multirow{2}{*}{ Pseudomonas spp. } & 2017 & 81 & & & & 29 & 29 & & & 79 & 90 & 76 & 48 & 47 & & 29 & \\
\hline & 2018 & 96 & & & & 50 & 49 & & & 85 & 94 & 67 & 70 & 85 & & 39 & \\
\hline
\end{tabular}

Figure 2 [The rate of antibiotics susceptibility among MDR-GNB isolates collected in 2017 AND 2018]. \%Susceptibility rate=Number of susceptible isolates/Total number of isolates $\times 100$. Boxes in green, yellow and red color reflect susceptibility rate of: $90-100 \%, 70-89 \%$ and $<70 \%$ respectively.

Abbreviations: AMIK, amikacin; AMP, ampicillin; AMC/CLAV, amoxicillin/clavulanic acid; CZO, cefazolin; FEB, cefepime; CAZ, ceftazidime; CRO, ceftriaxone; CXM, cefuroxime; CIP, ciprofloxacin; LEVO, levofloxacin; GENT, gentamicin; IMP, mipenem; MRP, meropenem; NFN, nitrofurantoin; TAZ, piperacillin/tazobactam; TRIMETH, trimethoprim.

( 926 of $1508,61.4 \%$ ) and $E$. coli isolates ( 812 of $1508,53.8 \%$ ). The most frequently isolated MDR-GNB were from patients between 20 and 29 years of age (211 of a total of 969, 21.8\%). Moreover, 643 (66.4\%) out of 969 MDR-GNBs were isolated from female patients, while 326 (33.6\%) were from male patients. ER had the highest rate of multidrug resistance among all other locations of sample collection (395 out of a total of 969, 40.8\%), followed by INP units (296 out of a total of $969,30.5 \%$ ). The majority of MDR-GNBs were from urine samples (593 of a total of 969, 60.2\%). E. coli ( 553 out of $969,57.1 \%$ ) had the highest rate of resistance among the isolated GNB, followed by K. pneumoniae (164 out of $969,16.9 \%)$ (Table 2).

Table 3 shows the association between the risk factors and MDR-GNBs included in the study. By including all sample types in the regression analysis, age was a positive and significant factor in the probability of having MDRGNB, with OR indicating that in every unit increase in this factor, the odds of MDR Gram-negative organisms change by a factor of 1.008 (95\% confidence interval [CI]: 1.004-1.012; $\mathrm{P}<0.001)$. The use of a central line (OR: $2.345,95 \%$ CI: $1.020-5.389$, P value: 0.045$)$, FC (OR: $1.475,95 \%$ CI: $1.044-2.084$, P value: 0.028$)$, mechanical ventilation (OR: 1.921, 95\% CI: $1.166-3.165$, P value: 0.010 ), history of operation during the 4 weeks before the onset of GNB (OR: $1.775,95 \%$ CI: $1.116-2.823$, P value: 0.015 ), and history of admission in SFHD during the past 6 months (OR: 1.721, $95 \%$ CI: $1.338-2.214$, P value: $<0.001$ ) were positive and significant predictors of the probability of developing MDRGNB according to the significant value. The INP units as a location of the sample collected in reference to the ER was a significant and positive factor of MDR-GNB (OR, 1.793; 95\% CI, 1.373-2.342; P value, 0.000). Sex and sample type were non-significant factors of MDR-GNB according to the significant values. When the risk factors were tested using specific sample risk factors (blood was used to test the central line, urine was used to test the FC, respiratory samples were used to test the mechanical ventilator, and wound and pus swabs were used to test the postoperative risk factor), the results showed that the use of a mechanical ventilator ( $\geq 72 \mathrm{~h}$ ) was a significant risk factor for the development of MDR-GNB (OR: 2.600, 95\% CI: 1.124-6.012, P value: 0.025). In contrast, the use of a central line $(\geq 72 \mathrm{~h})$ and $\mathrm{FC}(\geq 72 \mathrm{~h})$ and history of operation (past 4 weeks) were non-significant risk factors for the development of MDR-GNB. 
Table I Frequency of MDR GNB in Relation to Sociodemographic Features, and Risk Factors

\begin{tabular}{|c|c|c|c|}
\hline & 2017 & 2018 & Total \\
\hline & MDR-GNB n (\%) & MDR-GNB n (\%) & MDR-GNB n (\%) \\
\hline & $540(68.2)$ & 429 (59.9) & $969(64.3)$ \\
\hline \multicolumn{4}{|l|}{ Age groups } \\
\hline$>10$ & $84(65.1)$ & $63(50.4)$ & I 47 (57.9) \\
\hline $10-19$ & $31(55.4)$ & $25(55.6)$ & $56(55.4)$ \\
\hline $20-29$ & 137 (68.2) & $74(59.2)$ & $211(64.7)$ \\
\hline $30-39$ & $63(62.4)$ & $61(57.5)$ & $124(59.9)$ \\
\hline $40-49$ & $49(73.1)$ & $26(50)$ & $75(63)$ \\
\hline $50-59$ & $27(79.4)$ & $30(62.5)$ & $57(69.5)$ \\
\hline $60-69$ & $59(76.6)$ & $52(67.5)$ & III (72.I) \\
\hline 70-79 & 47 (79.7) & $39(75)$ & $86(77.5)$ \\
\hline $80-89$ & $32(59.3)$ & $45(67.2)$ & $77(63.6)$ \\
\hline$\leq 90$ & II (78.6) & 14 (73.7) & $25(75.8)$ \\
\hline \multicolumn{4}{|l|}{ Gender } \\
\hline Female & $377(68.1)$ & $266(56.6)$ & $643(62.8)$ \\
\hline Male & $163(68.5)$ & $163(66.3)$ & $326(67.4)$ \\
\hline \multicolumn{4}{|l|}{ Location } \\
\hline ICU & $51(67.1)$ & $29(63)$ & $80(65.6)$ \\
\hline INP & 145 (75.9) & $|5|(69.6)$ & $296(72.5)$ \\
\hline OP & $127(68.3)$ & $71(55)$ & $198(62.9)$ \\
\hline ER & $217(64)$ & I 78 (54.9) & $395(59.6)$ \\
\hline \multicolumn{4}{|l|}{ Sample } \\
\hline Urine & $338(68.4)$ & $255(59)$ & $593(64)$ \\
\hline Blood & $23(79.3)$ & $13(68.4)$ & $36(75)$ \\
\hline Respiratory & $37(72.5)$ & $29(54.7)$ & $66(63.5)$ \\
\hline Swab & $127(65.5)$ & $113(60.8)$ & $240(63.2)$ \\
\hline Others & $15(62.5)$ & $19(73.1)$ & $34(68)$ \\
\hline \multicolumn{4}{|c|}{ Gram-negative organisms } \\
\hline Acinetobacter spp. & $24(85.7)$ & $8(42.1)$ & $32(68.1)$ \\
\hline Enterobacter spp. & $29(80.6)$ & $35(92.1)$ & $64(86.5)$ \\
\hline Escherichia coli & $328(73.4)$ & $225(61.6)$ & $553(68.1)$ \\
\hline Klebsiella pneumoniae & $73(6 \mid .3)$ & $91(60.7)$ & $164(6 I)$ \\
\hline Proteus spp. & $42(67.7)$ & $42(77.8)$ & $84(72.4)$ \\
\hline
\end{tabular}

(Continued) 
Table I (Continued).

\begin{tabular}{|l|c|c|c|}
\hline Pseudomonas aeruginosa & $44(44)$ & $28(3 I . I)$ & $72(37.9)$ \\
\hline Risk factors & $21(80.8)$ & $8(80)$ & $29(80.6)$ \\
\hline Central line $(\geq 72 \mathrm{~h})$ & $71(74)$ & $56(69.1)$ & $127(71.8)$ \\
\hline Foley catheter ( $\geq 72 \mathrm{~h})$ & $47(8 \mathrm{I})$ & $23(69.7)$ & $70(76.9)$ \\
\hline Mechanical ventilator $(\geq 72 \mathrm{~h})$ & $44(77.2)$ & $33(73.3)$ & $77(75.5)$ \\
\hline Post-operation (past 4 weeks) & $153(80.1)$ & $139(66.5)$ & $292(64.3)$ \\
\hline History of admission in SFHD (past 6 months) & & & \\
\hline
\end{tabular}

To better understand the risk factors associated with multidrug resistance, an adjusted binary logistic regression was carried out using age and sex as adjustment variables. The use of a central line ( $\geq 72 \mathrm{~h})$ and FC ( $\geq 72 \mathrm{~h})$ and a history of operation during the 4 weeks before the onset of MDR-GNB were not statistically significant risk factors. However, age (OR: 1.007, 95\% CI: $1.003-1.012$, P value: 0.001 ), use of a mechanical ventilator ( $\geq 72 \mathrm{~h}$; OR: 1.671 , 95\% CI: $1.002-2.785$, P value: 0.049 ), history of admission to SFHD during the past 6 months (OR: $1.607,95 \%$ CI: 1.244-2.076, P value: $<0.001$ ), and collection of samples from the INP units (OR: 1.584, 95\% CI: 1.189-2.112, P = 0.002) were still considered statistically significant risk factors. Binary logistic regression was performed using age, sex, and location of sample collection as adjustment variables. Age (OR: 1.005, 95\% CI: 1.001-1.010, P = 0.013), use of a central line $(\geq 72 \mathrm{~h})(\mathrm{OR}: 2.521,95 \% \mathrm{CI}: 1.028-6.182, \mathrm{P}=0.043)$ and mechanical ventilation $(\geq 72 \mathrm{~h})(\mathrm{OR}$ : 2.218, 95\% CI: $1.189-4.135, \mathrm{P}=0.012$ ), and history of admission to SFHD during the past 6 months (OR: 1.558 , 95\% CI: $1.203-2.019, \mathrm{P}=0.001$ ) were statistically significant risk factors for the development of MDR-GNB. Furthermore, it was found that the respiratory (OR: 0.606, 95\% CI: 0.368-0.999, P value: 0.050 ) and swab (OR: $0.756,95 \%$ CI: $0.572-0.998$, P value: 0.048 ) samples were statistically significant predictors of MDR-GNB (Table 3 ).

\section{Discussion}

In this study, we aimed to determine the prevalence of MDROs among the GNB isolated from various departments of the SFHD during 2017 and 2018 and to identify the associated risk factors. It was found that the prevalence of MDR-GNB in 2017 was higher than that in 2018 (68.2\% and 59.9\%, respectively), and the overall rate during the two years was $64.3 \%$. The observed decline in the resistance rate in 2018 was largely related to the increased antibiotic stewardship interventions of the SFHD in that year compared to those undertaken in 2017. Our finding is similar to that of the study by UcCachón et al, where the authors found that $70.96 \%$ of GNB isolated from ICU, pediatric ICU, and neonatal ICU patients were MDR. ${ }^{16}$ In contrast, in another retrospective study conducted from July 1, 2013, to July 31, 2014, at Saint Louis University Hospital in Missouri, USA, 26\% of the GNB included in the study were MDR. ${ }^{6}$

In a study conducted by Patolia et al, only patients aged 18 years and above were included. ${ }^{6}$ In comparison, we considered all age groups, which may explain the higher rate of multidrug resistance observed in our study, given that high numbers of MDR-GNB were isolated from our participants who were less than 18 years of age. In addition, the most frequently isolated MDR-GNB in our study was E. coli, which is consistent with the results (38\% prevalence) reported by Patolia et al. ${ }^{6}$ However, some other studies have reported other MDR-GNB to exhibit the highest prevalence rate, such Acinetobacter spp. (40.4\%) reported by a study conducted on the ICU patients of Riyadh Military Hospital in 2010. ${ }^{17}$ This result was also corroborated by a study conducted at the two largest public-sector hospitals in Singapore from 2007 to 2009, in which the most frequently isolated MDR-GNB were Acinetobacter spp. $(14.3 \%),{ }^{17}$ as well as by another study conducted in Sultan Qaboos University Hospital in Oman in 2012 in which also Acinetobacter spp. were the most frequently isolated MDR bacteria among the GNB included in the study (32.4\%). ${ }^{17}$ In our research, MDR Acinetobacter spp. constituted the least frequently isolated organisms among all 
Table 2 Descriptive Characteristics of MDR-GNB Over the Study Period in Relation to Patients' Sociodemographic Features and Health Indicators

\begin{tabular}{|c|c|c|c|c|c|c|}
\hline & \multicolumn{2}{|c|}{2017} & \multicolumn{2}{|c|}{2018} & \multicolumn{2}{|c|}{ Total } \\
\hline & No. of GNB & $\begin{array}{c}\text { MDR-GNB } \\
\text { n (\%) }\end{array}$ & No. of GNB & $\begin{array}{c}\text { MDR-GNB } \\
\text { n (\%) }\end{array}$ & No. of GNB & $\begin{array}{c}\text { MDR-GNB } \\
n(\%)\end{array}$ \\
\hline & 792 & $540(100)$ & 716 & $429(100)$ & 1508 & $969(100)$ \\
\hline $\begin{array}{l}\text { Age } \\
\text { groups }\end{array}$ & \multicolumn{2}{|c|}{$\left(X^{2}=17.899, d f=9, P\right.$ value $\left.=0.036\right)$} & \multicolumn{2}{|c|}{$\left(X^{2}=17.358, d f=9, P\right.$ value $\left.=0.043\right)$} & \multicolumn{2}{|c|}{$\begin{array}{c}\left(X^{2}=25.193, d f=9\right. \\
P \text { value }=0.003)\end{array}$} \\
\hline$>10$ & 129 & $84(15.6)$ & 125 & $63(14.7)$ & 245 & $147(15.2)$ \\
\hline $10-19$ & 56 & 31 (5.7) & 45 & $25(5.8)$ & 101 & $56(5.8)$ \\
\hline $20-29$ & 201 & $137(25.4)$ & 125 & $74(17.2)$ & 326 & $211(21.8)$ \\
\hline $30-39$ & 101 & $63(11.7)$ & 106 & $61(14.2)$ & 207 & $124(12.8)$ \\
\hline $40-49$ & 67 & $49(9.1)$ & 52 & $26(6.1)$ & 119 & $75(7.7)$ \\
\hline $50-59$ & 34 & $27(5)$ & 48 & $30(7)$ & 82 & $57(5.9)$ \\
\hline $60-69$ & 77 & $59(10.9)$ & 77 & $52(12.1)$ & 154 & III (II.5) \\
\hline 70-79 & 59 & $47(8.7)$ & 52 & $39(9.1)$ & 111 & $86(8.9)$ \\
\hline $80-89$ & 54 & $32(5.9)$ & 66 & $45(10.5)$ & 121 & $77(7.9)$ \\
\hline$\leq 90$ & 14 & II (2) & 20 & $14(3.3)$ & 33 & $25(2.6)$ \\
\hline Gender & \multicolumn{2}{|c|}{$\left(X^{2}=0.015, d f=I, P\right.$ value $\left.=0.904\right)$} & \multicolumn{2}{|c|}{$\left(X^{2}=6.280, d f=I, P\right.$ value $\left.=0.012\right)$} & \multicolumn{2}{|c|}{$\begin{array}{c}\left(X^{2}=2.979, d f=I,\right. \\
P \text { value }=0.084)\end{array}$} \\
\hline Female & 554 & $377(69.8)$ & 470 & $266(62)$ & 1024 & $643(66.4)$ \\
\hline Male & 238 & $163(30.2)$ & 246 & $163(38)$ & 484 & $326(33.6)$ \\
\hline Location & \multicolumn{2}{|c|}{$\left(X^{2}=8.025, d f=3, P\right.$ value $\left.=0.045\right)$} & \multicolumn{2}{|c|}{$\left(X^{2}=13.255, d f=3, P\right.$ value $\left.=0.004\right)$} & \multicolumn{2}{|c|}{$\left(X^{2}=18.896, d f=3, P\right.$ value $\left.=0.000\right)$} \\
\hline ICU & 76 & $51(9.4)$ & 46 & $29(6.8)$ & 122 & $80(8.3)$ \\
\hline INP & 191 & $145(26.9)$ & 217 & $|5|$ (35.2) & 408 & $296(30.5)$ \\
\hline OP & 186 & $127(23.5)$ & 129 & 71 (16.6) & 315 & $198(20.4)$ \\
\hline ER & 339 & $217(40.2)$ & 324 & I78 (4I.5) & 663 & $395(40.8)$ \\
\hline
\end{tabular}




\begin{tabular}{|c|c|c|c|c|c|c|}
\hline \multirow{2}{*}{$\begin{array}{l}\text { Sample } \\
\text { Urine }\end{array}$} & \multicolumn{2}{|c|}{$\left(X^{2}=3.135, d f=4, P\right.$ value $\left.=0.536\right)$} & \multicolumn{2}{|c|}{$\left(X^{2}=3.240, d f=4, P\right.$ value $\left.=0.518\right)$} & \multicolumn{2}{|c|}{$\left(X^{2}=2.965, d f=4, P\right.$ value $\left.=0.564\right)$} \\
\hline & 494 & $338(62.6)$ & 432 & $255(59.4)$ & 926 & $593(61.2)$ \\
\hline Blood & 29 & $23(4.3)$ & 19 & $13(3)$ & 48 & $36(3.7)$ \\
\hline Respiratory & 51 & $37(6.9)$ & 53 & $29(6.8)$ & 104 & $66(6.8)$ \\
\hline Sputum & 12 & 8 & 13 & 3 & 25 & 11 \\
\hline Tracheal aspirate & 38 & 28 & 36 & 23 & 74 & 51 \\
\hline Bronchial lavage and washing & 1 & 1 & 3 & 2 & 4 & 3 \\
\hline Sinus aspirate & - & - & I & 1 & 1 & 1 \\
\hline Swab & 194 & $127(23.5)$ & 186 & $113(26.3)$ & 380 & $240(24.8)$ \\
\hline Anal & 1 & 1 & 2 & 2 & 3 & 3 \\
\hline Ear & 20 & 5 & 26 & 6 & 46 & 11 \\
\hline Endometrial & 1 & - & - & - & 1 & - \\
\hline Eye & 5 & 3 & 6 & 2 & 11 & 5 \\
\hline Genital & 1 & 1 & - & - & 1 & 1 \\
\hline Groin & 2 & 1 & 2 & 2 & 4 & 3 \\
\hline Placental & - & - & 2 & - & 2 & - \\
\hline Pus & 105 & 78 & 108 & 75 & 213 & 153 \\
\hline Skin & - & - & 3 & 3 & 3 & 3 \\
\hline Throat & 2 & - & 3 & - & 5 & - \\
\hline Vulval & 2 & - & - & - & 2 & - \\
\hline Wound & 55 & 38 & 34 & 23 & 89 & 61 \\
\hline Others & 24 & $15(2.8)$ & 26 & $19(4.4)$ & 50 & $34(3.5)$ \\
\hline Bile Fluid & 1 & 1 & - & - & 1 & 1 \\
\hline Catheter Tip & 8 & 4 & 10 & 8 & 18 & 12 \\
\hline
\end{tabular}


Table 2 (Continued).

\begin{tabular}{|c|c|c|c|c|c|c|}
\hline Peritoneal Fluid & I & 1 & 1 & 1 & 2 & 2 \\
\hline Semen & 3 & $\mathrm{I}$ & - & - & 3 & 1 \\
\hline Tissue & 11 & 8 & 15 & 10 & 26 & 18 \\
\hline Gram-negative organisms & \multicolumn{2}{|c|}{$\left(X^{2}=41.596, d f=5, P\right.$ value $\left.=0.000\right)$} & \multicolumn{2}{|c|}{$\left(X^{2}=57.659, d f=5, P\right.$ value $\left.=0.000\right)$} & \multicolumn{2}{|c|}{$\left(X^{2}=83.573, d f=5, P\right.$ value $\left.=0.000\right)$} \\
\hline Acinetobacter spp. & 28 & $24(4.4)$ & 19 & $8(1.9)$ & 47 & $32(3.3)$ \\
\hline Enterobacter spp. & 36 & $29(5.4)$ & 38 & $35(8.2)$ & 74 & $64(6.6)$ \\
\hline Escherichia coli & 447 & $328(60.7)$ & 365 & $225(52.4)$ & 812 & $553(57.1)$ \\
\hline Klebsiella pneumoniae & 119 & $73(13.5)$ & 150 & $91(21.2)$ & 269 & $164(16.9)$ \\
\hline Proteus spp. & 62 & $42(7.8)$ & 54 & $42(9.8)$ & 116 & $84(8.7)$ \\
\hline Pseudomonas aeruginosa & 100 & $44(8.1)$ & 90 & $28(6.5)$ & 190 & $72(7.4)$ \\
\hline \multicolumn{7}{|l|}{ Risk factors } \\
\hline Central line $(\geq 72 \mathrm{~h})$ & 26 & 21 & 10 & 8 & 36 & 29 \\
\hline Foley catheter $(\geq 72 \mathrm{~h})$ & 96 & 71 & 81 & 56 & 177 & 127 \\
\hline Mechanical ventilator $(\geq 72 \mathrm{~h})$ & 58 & 47 & 33 & 23 & 91 & 70 \\
\hline Post-operation (past 4 weeks) & 57 & 44 & 45 & 33 & 102 & 77 \\
\hline History of admission in SFHD (past 6 months) & 191 & 153 & 209 & 139 & 400 & 292 \\
\hline
\end{tabular}


Table 3 Logistic Regression Analysis of Risk Factors for MDR GNB

\begin{tabular}{|c|c|c|c|c|c|c|c|c|c|c|c|c|c|}
\hline \multirow[t]{3}{*}{ Risk Factors } & \multirow[t]{3}{*}{ Categories } & \multicolumn{4}{|c|}{ Unadjusted } & \multicolumn{4}{|c|}{ Adjusted $^{\mathrm{a}}$} & \multicolumn{4}{|c|}{ Adjusted $^{\mathbf{b}}$} \\
\hline & & \multirow[t]{2}{*}{ OR } & \multicolumn{2}{|c|}{$95 \% \mathrm{Cl}$} & \multirow[t]{2}{*}{$P$ val. } & \multirow[t]{2}{*}{ OR } & \multicolumn{2}{|c|}{$95 \% \mathrm{Cl}$} & \multirow[t]{2}{*}{$P$ val. } & \multirow[t]{2}{*}{ OR } & \multicolumn{2}{|c|}{$95 \% \mathrm{Cl}$} & \multirow[t]{2}{*}{$P$ val. } \\
\hline & & & Lower & Upper & & & Lower & Upper & & & Lower & Upper & \\
\hline \multicolumn{14}{|l|}{ All samples } \\
\hline Age & Years & 1.008 & 1.004 & 1.012 & 0.000 & 1.007 & 1.003 & 1.012 & 0.001 & 1.006 & 1.001 & 1.010 & 0.013 \\
\hline \multirow[t]{2}{*}{ Gender } & Female & \multicolumn{12}{|l|}{ Ref. } \\
\hline & Male & 1.223 & 0.973 & 1.536 & 0.085 & 1.089 & 0.858 & 1.382 & 0.485 & 1.023 & 0.798 & 1.312 & 0.857 \\
\hline \multirow[t]{2}{*}{ Central line $(\geq 72 \mathrm{~h})$} & No & \multicolumn{12}{|l|}{ Ref. } \\
\hline & Yes & 2.345 & 1.020 & 5.389 & 0.045 & 2.167 & 0.936 & 5.016 & 0.071 & 2.521 & 1.028 & 6.182 & 0.043 \\
\hline \multirow[t]{2}{*}{ Foley catheter $(\geq 72 \mathrm{~h})$} & No & \multicolumn{12}{|l|}{ Ref. } \\
\hline & Yes & 1.475 & 1.044 & 2.084 & 0.028 & 1.156 & 0.792 & 1.686 & 0.453 & 1.066 & 0.702 & 1.621 & 0.764 \\
\hline \multirow[t]{2}{*}{ Mechanical ventilator $(\geq 72 \mathrm{~h})$} & No & \multicolumn{12}{|l|}{ Ref. } \\
\hline & Yes & 1.921 & 1.166 & 3.165 & 0.010 & 1.671 & 1.002 & 2.785 & 0.049 & 2.218 & 1.189 & 4.135 & 0.012 \\
\hline \multirow[t]{2}{*}{ Post-operation (past 4 weeks) } & No & \multicolumn{12}{|l|}{ Ref. } \\
\hline & Yes & 1.775 & 1.116 & 2.823 & 0.015 & 1.595 & 0.996 & 2.554 & 0.052 & 1.430 & 0.887 & 2.306 & 0.143 \\
\hline \multirow[t]{2}{*}{ History of admission in SFHD (past 6 months) } & No & \multicolumn{12}{|l|}{ Ref. } \\
\hline & Yes & 1.721 & 1.338 & 2.214 & 0.000 & 1.607 & 1.244 & 2.076 & 0.000 & 1.558 & 1.203 & 2.019 & 0.001 \\
\hline \multirow[t]{5}{*}{ Samples } & Urine & \multicolumn{12}{|l|}{ Ref. } \\
\hline & Blood & 1.685 & 0.865 & 3.282 & 0.125 & 1.500 & 0.765 & 2.943 & 0.238 & 1.354 & 0.676 & 2.715 & 0.393 \\
\hline & Respiratory & 0.975 & 0.640 & 1.486 & 0.907 & 0.742 & 0.473 & 1.163 & 0.193 & 0.606 & 0.368 & 0.999 & 0.050 \\
\hline & Swab & 0.963 & 0.751 & 1.233 & 0.764 & 0.865 & 0.663 & 1.128 & 0.284 & 0.756 & 0.572 & 0.998 & 0.048 \\
\hline & Others & 1.193 & 0.649 & 2.194 & 0.570 & 0.932 & 0.497 & 1.747 & 0.825 & 0.708 & 0.369 & 1.356 & 0.298 \\
\hline
\end{tabular}


Table 3 (Continued).

\begin{tabular}{|c|c|c|c|c|c|c|c|c|c|c|c|c|c|}
\hline \multirow[t]{3}{*}{ Risk Factors } & \multirow[t]{3}{*}{ Categories } & \multicolumn{4}{|c|}{ Unadjusted } & \multicolumn{4}{|c|}{ Adjusted $^{\mathrm{a}}$} & \multicolumn{4}{|c|}{ Adjusted $^{\mathrm{b}}$} \\
\hline & & \multirow[t]{2}{*}{ OR } & \multicolumn{2}{|c|}{$95 \% \mathrm{Cl}$} & \multirow[t]{2}{*}{$P$ val. } & \multirow[t]{2}{*}{ OR } & \multicolumn{2}{|c|}{$95 \% \mathrm{Cl}$} & \multirow[t]{2}{*}{$P$ val. } & \multirow[t]{2}{*}{ OR } & \multicolumn{2}{|c|}{$95 \% \mathrm{Cl}$} & \multirow[t]{2}{*}{$P$ val. } \\
\hline & & & Lower & Upper & & & Lower & Upper & & & Lower & Upper & \\
\hline \multirow[t]{4}{*}{ Location } & ER & \multicolumn{12}{|c|}{ Ref. } \\
\hline & ICU & 1.292 & 0.862 & 1.936 & 0.214 & 1.054 & 0.676 & 1.643 & 0.815 & & & & \\
\hline & INP & 1.793 & 1.373 & 2.342 & 0.000 & 1.584 & 1.189 & 2.112 & 0.002 & & & & \\
\hline & OP & 1.148 & 0.871 & 1.513 & 0.327 & 1.089 & 0.823 & 1.441 & 0.550 & & & & \\
\hline \multicolumn{14}{|l|}{ Specific samples } \\
\hline Central line $(\geq 72 \mathrm{~h})$ & Blood & 1.667 & 0.306 & 9.080 & 0.555 & 2.725 & 0.412 & 18.041 & 0.299 & 2.773 & 0.261 & 29.447 & 0.398 \\
\hline Foley catheter ( $\geq 72 \mathrm{~h}$ ) & Urine & 1.343 & 0.708 & 2.547 & 0.367 & 1.009 & 0.505 & 2.015 & 0.981 & 0.976 & 0.458 & 2.080 & 0.950 \\
\hline Mechanical ventilator $(\geq 72 \mathrm{~h})$ & Respiratory & 2.600 & 1.124 & 6.012 & 0.025 & 2.682 & 1.149 & 6.261 & 0.022 & 2.224 & 0.868 & 5.703 & 0.096 \\
\hline Post-operation (past 4 weeks) & Wound and pus swab & 1.296 & 0.679 & 2.471 & 0.431 & 1.321 & 0.691 & 2.524 & 0.400 & 1.327 & 0.690 & 2.552 & 0.397 \\
\hline
\end{tabular}

Notes: a Adjusted logistic regression (age and gender). ${ }^{b}$ Adjusted logistic regression (age, gender, and location of sample collection).

Abbreviations: AMR, antimicrobial- resistance; E. coli, Escherichia coli; ER, emergency room; ESBL, extended-spectrum beta-lactamases; GNB, Gram-negative bacteria; HIS, hospital information system; ICU, intensive care unit; INP, inpatient; IRB, Institutional Review Board; K. pneumoniae, Klebsiella pneumoniae; LIS, laboratory information system; MDR, multidrug-resistant; MDR-GNB, multidrug-resistant Gram-negative bacteria; MDROs, multidrug-resistant organisms; NICU, neonatal intensive care unit; OB/GYN, obstetrics/gynecology; OP, outpatient; P. aeruginosa, Pseudomonas aeruginosa; PICU, pediatric intensive care unit; SFHD, Security Forces Hospital-Dammam. 
MDR-GNB identified in the study. This may be because we included patients from all hospital departments—not only inpatients (INPs) — and from different age groups, including pediatric patients. In addition, most of our samples were urine specimens, similar to those utilized by O'Fallon et al; in their study, 93.9\% of MDR-GNB were from urine samples. ${ }^{18}$

Regarding the MDR-GNB distribution among male and female patients, we found that out of the 969 patients from whom MDR-GNB were isolated, the bacteria were more frequently isolated from female patients, while in the study by Lye et al. ${ }^{19}$ male patients showed a higher prevalence (57\%) of MDR-GNB. This may be because we also included the $\mathrm{OB} / \mathrm{GYN}$ ward (which consists only of female patients) in our sample selection criteria, as a result of which, more female patients were involved, increasing the possibility of MDR-GNB isolation from this cohort group. In addition, Lye et al limited their sample selection criteria to participants who developed sepsis due to infection with either $P$. aeruginosa or A. baumannii. ${ }^{19}$ In contrast, we included all patients who provided specimens during the study period.

The lowest prevalence rate of MDR-GNB was among patients aged less than 9 years, while the highest was among the patients aged between 70 and 79 years, followed by patients aged $>90$ years, as the risk of having MDR-GNB increases by a factor of 1.008 for each year of age. Although the estimated risk appears minimal, with extreme age (eg, 70 years), this risk would elevate dramatically, especially when considering that elderly patients may be prone to frequent hospitalization and have weak immunity. Accordingly, serious complications may occur once MDR- GNBs enter their bloodstream and cannot be treated.

Although Enterobacter spp. were not the most commonly reported MDR-GNB among our isolates, most of the isolated Enterobacter spp. were resistant to multiple drugs. The study by Uc-Cachón et al. ${ }^{16}$ reported $E$. coli to show the highest resistance (91.57\%) among MDR-GNB. On the other hand, Al Yousef (2016) reported P. aeruginosa to show the highest rate of resistance as approximately $100 \%$ of the isolated $P$. aeruginosa were MDR. ${ }^{20}$ In the study by Cachón et al, only ICU patients were included, and the majority of their samples were from the bronchial aspirate of the patients, while in the study by Al Yousef (2016), wound samples were included; this was limited in our data set. Our study found that the rate of multidrug resistance was high among the GNB isolated from blood samples.

Regarding our regression analysis results to identify the risk factors for the emergence and growth of MDR-GNB, our findings were aligned with the results reported by Patolia et al, who identified urinary catheters as a significant risk factor for the emergence and growth of MDR-GNB. ${ }^{6}$ However, they also found that using a central line and mechanical ventilator was not a significant risk factor, which differs from our results, which we obtained by including all our participants in the regression models regardless of their sample type. However, when the regression analyses were repeated using only subgroups based on their specific sample type to estimate the risk of a particular risk factor, the use of a mechanical ventilator remained statistically significant. In contrast, the use of a central line was not statistically significant, as was the use of FC. This may indicate a complication of the relationship between the chosen risk factors and MDR-GNB, as other latent unmeasured factors may play an essential role in determining the significance of this relationship. For example, unlike the study of Patolia et al, which included only samples collected from adult hospitalized patients, our samples were also obtained from outpatients; hence, having this group among our participants may have confounded the results of our analysis. Although we adjusted our regression models for the location of sample collection variables, the use of a mechanical ventilator and central line for three calendar days or more remained a statistically significant risk factor, as we included all study specimens regardless of the sample type in the analysis. However, this risk factor became statistically insignificant when we carried out the regression analysis using a specific subgroup based on the sample types for each risk factor. This difference in results following adjustment for confounders and stratification may support the role of confounding and mediation, as well as the possibility of there being other unmeasured confounders (eg, history of recurrent antibiotics) and characteristics of our hospital (eg, protocols of antibiotic prescription) or involved patients (eg, their chief complaints) that affected our results.

In this context, the type of the collected samples, which by itself was not considered a direct risk factor for the emergence and growth of MDR-GNB, was included in the regression analysis to represent the unmeasured factors associated with MDR-GNB that were not included in prior analysis, such as the possible source of current infection or the protocol of sample collection. The results of the regression analysis adjusted for the location of the samples stratified by age and sex showed that respiratory and swab samples were significantly associated with MDR-GNB, which may 
indicate that respiratory, ear, eye, anal, endometrial, genital, groin, placental, pus, skin, throat, vulval, or wound infections were significant contributors to the development of MDR-GNB.

Our results also showed that a history of operation during the four weeks before the onset of MDR-GNB infection and a history of admission in the SFHD during the past six months were statistically significant and possible predictors of MDR-GNB infection. This finding may be due to the increased risk of acquiring a drug-resistant nosocomial infection with recurrent hospital admission, as several antimicrobial drugs are prescribed daily in the hospital setting. Supporting this idea, when the location of sample collection was included in the analysis using the ER as a reference, the results showed that samples extracted and collected from the INP units were a statistically significant predictor of MDR-GNB. In contrast, those of the OP units were not statistically significant. However, other unstudied factors influencing this relationship (between location of sample collection and MDR-GNB) may have affected this result, such as the length of hospital stay and the type of disease experienced before the onset of MDR-GNB infection, which may explain why the ICU setting was not a risk factor for the development of MDR-GNB. Sample type was not found to be a statistically significant predictor of MDR GNB infection. However, when the regression analysis was repeated using subgroups based on the type of samples, we found that the use of a mechanical ventilator for $72 \mathrm{~h}$ or more was a significant and positive risk factor for the development of MDR-GNB, while the use of a central line and FC and post-operation were nonsignificant risk factors according to the significance level, which was more than 0.05. Although we obtained these adjusted regression analysis results for age, sex, and sample location by grouping, the results may still be influenced by various confounders that we could not report or measure in this study due to unavailability in the HIS or LIS database, such as history of admission to another hospital, previous history of misuse of antibiotics, and the length of hospital stay. Additionally, our study findings may differ in some aspects from other studies, as the SFHD is a secondary care hospital, whereas most of the studies were conducted in a tertiary care hospital with critically ill patients who may receive further advanced treatments. These patients may be considered to be at a greater risk of acquiring an infection or becoming infected with various strains of nosocomial MDR-GNB, especially in units such as cancer and burn units, and may be provided advanced care to prevent such an infection.

Although this was a comprehensive study, it has several limitations. First, there were various factors that we could not access and could not report their effects as risk factors for the development of MDR-GNB infection, such as comorbidity, history and pattern of antibiotic usage, and length of hospital stay before the onset of an MDR GNB infection. In addition, using a single center (ie, the SFHD) may limit the generalizing of our results to patients of other hospitals because of the distinctiveness of our patients, the specific hospital management protocols, and the infection control measures. A plan for future continued research could involve comparing our findings with those of similar studies undertaken in a referral or a tertiary hospital in our region. Additionally, evaluating the risk factors at the species level and not just the genus level (P. mirabilis relative to the other Proteus species) may help flag bacterial species with clinical relevance.

\section{Conclusion}

In conclusion, in the current study, we found a high prevalence of MDR-GNBs, with an overall rate of $64.3 \%$. It has been demonstrated that using a mechanical ventilator for three or more calendar days is a significant direct risk factor for the development of MDR-GNBs. Therefore, we must emphasize and strengthen the role of antimicrobial stewardship programs in hospitals. The awareness of healthcare workers on MDR-GNBs and preventive measures is highly recommended and required. Raising public and medical attention and knowledge of the proper use of antibiotics will help prevent the acquisition of MDROs. ${ }^{21}$

\section{Data Sharing Statement}

The datasets used and analyzed during the current study are available from the corresponding author upon reasonable request.

\section{Ethics Approval and Consent to Participate}

This study was approved by the Institutional Review Board (IRB) committee of Imam Abdulrahman Bin Faisal University (IRB-PGS-2020-03-005). In addition, approval was obtained from the SFHD, transaction number 
(41102069, 27th January 2020). The requirement for informed consent was waived by the IRB committee as it involved data collected from laboratory databases and infection control records for the purpose of research. Data were used confidentially and were saved in a password-protected computer. Patients included in the study were anonymous; neither ID nor personally identifiable information, such as name or home address, was used. This study was conducted in accordance with the Declaration of Helsinki.

\section{Acknowledgments}

The authors would like to thank all members of the Diagnostic Microbiology Laboratory and Infection Control Department at the Security Forces Hospital, Dammam, for their help with the data collection.

\section{Author Contributions}

All authors made substantial contributions to the conception and design, acquisition of data, analysis and interpretation of data, drafting the article or revising it critically for important intellectual content, agreed to submit to the current journal, provided final approval of the version to be published, and agreed to be accountable for all aspects of the work.

\section{Disclosure}

The authors declare that they have no competing interests in this work.

\section{References}

1. Blair JM, Webber MA, Baylay AJ, Ogbolu DO, Piddock LJ. Molecular mechanisms of antibiotic resistance. Nat Rev Microbiol. $2015 ; 13(1)$ :42-51. doi:10.1038/nrmicro3380

2. Hall KK, Shoemaker-Hunt S, Hoffman L, et al. Making healthcare safer iii: a critical analysis of existing and emerging patient safety practices. Agency for Healthcare Research and Quality (US); 2020. Available from: https://www.ncbi.nlm.nih.gov/books/NBK555533/. Accessed February 4, 2022.

3. Rodríguez-Baño J, Gutiérrez-Gutiérrez B, Machuca I, Pascual A. Treatment of infections caused by extended-spectrum-beta-lactamase-, AmpC-, and carbapenemase-producing Enterobacteriaceae. Clin Microbiol Rev. 2018;31(2). doi:10.1128/CMR.00079-17

4. Antimicrobial resistance: tackling a crisis for the health and wealth of nations. London: Wellcome Trust; 2014.

5. Dadgostar P. Antimicrobial resistance: implications and costs. Infect Drug Resist. 2019;12:3903-3910. doi:10.2147/IDR.S234610

6. Patolia S, Abate G, Patel N, Frey S. Risk factors and outcomes for multidrug-resistant Gram-negative bacilli bacteremia. Ther Adv Infect Dis. 2018;5(1):11-18. doi:10.1177/2049936117727497

7. Gogry FA, Siddiqui MT, Sultan I, Haq QMR. Current update on intrinsic and acquired colistin resistance mechanisms in bacteria. Front Med. 2021;8:677720. doi:10.3389/fmed.2021.677720

8. Alamri AM, Alsultan AA, Ansari MA, Alnimr AM. Biofilm-formation in clonally unrelated multidrug-resistant. Pathogens. 2020;9(8). doi:10.3390/pathogens 9080630

9. Yamani L, Alamri A, Alsultan A, Alfifi S, Ansari MA, Alnimr A. Inverse correlation between biofilm production efficiency and antimicrobial resistance in clinical isolates of Pseudomonas aeruginosa. Microb Pathog. 2021;157:104989. doi:10.1016/j.micpath.2021.104989

10. Ventola CL. The antibiotic resistance crisis: part 1: causes and threats. $P$ T. 2015;40(4):277-283.

11. Zowawi HM. Antimicrobial resistance in Saudi Arabia: an urgent call for an immediate action. Saudi Med J. 2016;37(9):935-940. doi:10.15537/ smj.2016.9.16139

12. AlAmri AM, AlQurayan AM, Sebastian T, AlNimr AM. Molecular surveillance of multidrug-resistant Acinetobacter baumannii. Curr Microbiol. 2020;77(3):335-342. doi:10.1007/s00284-019-01836-Z

13. Al Mayahi Z, Kamel S, Amer H, Beatty M. Outbreak of colistin-resistant organisms at a tertiary hospital in Riyadh, Saudi Arabia, 2016. Pan Afr Med J. 2019;34:162. doi:10.11604/pamj.2019.34.162.19998

14. Magiorakos AP, Srinivasan A, Carey RB, et al. Multidrug-resistant, extensively drug-resistant and pandrug-resistant bacteria: an international expert proposal for interim standard definitions for acquired resistance. Clin Microbiol Infect. 2012;18(3):268-281. doi:10.1111/j.14690691.2011.03570.x

15. Clinical and Laboratory Standards Institute. The Clinical Laboratory Standards Institute, Performance Standards for Antimicrobial Susceptibility Testing. 28th ed. Vol. 27. Wayne, NJ, USA: Clinical and Laboratory Standards Institute; 2018.

16. Uc-Cachón AH, Gracida-Osorno C, Luna-Chi IG, Jiménez-Guillermo JG, Molina-Salinas GM. High prevalence of antimicrobial resistance among gram-negative isolated bacilli in intensive care units at a tertiary-care hospital in Yucatán Mexico. Medicina. 2019;55(9). doi:10.3390/ medicina55090588

17. Saeed NK, Kambal AM, El-Khizzi NA. Antimicrobial-resistant bacteria in a general intensive care unit in Saudi Arabia. Saudi Med J. 2010;31 (12):1341-1349.

18. O'Fallon E, Pop-Vicas A, D'Agata E. The emerging threat of multidrug-resistant Gram-negative organisms in long-term care facilities. $J$ Gerontol a Biol Sci Med Sci. 2009;64(1):138-141. doi:10.1093/gerona/gln020

19. Lye DC, Earnest A, Ling ML, et al. The impact of multidrug resistance in healthcare-associated and nosocomial Gram-negative bacteraemia on mortality and length of stay: cohort study. Clin Microbiol Infect. 2012;18(5):502-508. doi:10.1111/j.1469-0691.2011.03606.x 
20. Al Yousef SA. Surveillance of antibiotic-resistant bacteria in king khalid hospital, Hafr Al-Batin, Saudi Arabia, during 2013. Jundishapur J Microbiol. 2016;9(9):e19552. doi:10.5812/jjm.19552

21. Centers for Disease Control and Prevention. Core elements of antibiotic stewardship; 2021.

\section{Publish your work in this journal}

Infection and Drug Resistance is an international, peer-reviewed open-access journal that focuses on the optimal treatment of infection (bacterial, fungal and viral) and the development and institution of preventive strategies to minimize the development and spread of resistance. The journal is specifically concerned with the epidemiology of antibiotic resistance and the mechanisms of resistance development and diffusion in both hospitals and the community. The manuscript management system is completely online and includes a very quick and fair peer-review system, which is all easy to use. Visit http://www.dovepress.com/testimonials.php to read real quotes from published authors.

Submit your manuscript here: https://www.dovepress.com/infection-and-drug-resistance-journal 